\title{
Integration of mechanical and chemical signals by YAP and TAZ transcription coactivators
}

\author{
Xiaocan Guo and Bin Zhao*
}

\begin{abstract}
YAP and TAZ are transcription coactivators and effectors of the Hippo pathway, which play a key role in organ size control. Through interaction with transcription factors such as TEADs, they activate gene transcription and thus promote cell proliferation, inhibit apoptosis, and regulate cell differentiation. Dysregulation of YAP/TAZ was found to correlate with human cancers. The oncogenic roles of these proteins were also demonstrated in animal models. The growth promoting activity of YAP/TAZ is limited by the Hippo tumor suppressor pathway through phosphorylation-induced cytoplasmic retention and destabilization. Recently, it was found that YAP and TAZ mediate responses to several extracellular signals including mechanical stress, GPCR signaling, and the Wnt signaling pathway. All these growth-regulating signals play important roles in normal development and cancer. In this review, we would like to discuss the function of YAP and TAZ as effectors of these physiological signals.
\end{abstract}

Keywords: YAP, TAZ, The Hippo pathway, mechanical stress, GPCR signaling, Wnt pathway

\section{Introduction}

The precise control of cell number during development and regeneration maintains organ size homeostasis of multicellular organisms. In recent years, the Hippo signaling pathway is emerging as a key regulator of organ size $[1,2]$. The Hippo pathway was first identified in Drosophila by genetic mosaic screens for growth inhibitory genes [3-12]. Then the pathway and its function in organ size control were found to be conserved in mammals $[13,14]$. Yes-associated protein (YAP) and its homolog transcriptional co-activator with PDZ-binding motif (TAZ, also called WWTR1) are key downstream effectors of the Hippo pathway being phosphorylated and inhibited by the Hippo pathway kinases Last $1 / 2$ $[13,15]$. YAP and TAZ activate gene transcription through interaction with transcription factors such as the four TEAD family proteins [16,17]. YAP/TAZ-induced gene expression results in cell proliferation, evasion of apoptosis, and also amplification of progenitor/stem cells thus promotion of organ size. Consistently, recent studies on animal models also demonstrate a role of the Hippo pathway and YAP in tissue regeneration [18,19]. Furthermore, there are ever-accumulating reports on the correlation of

\footnotetext{
* Correspondence: binzhao@zju.edu.cn

Life Sciences Institute, Zhejiang University, Hangzhou, Zhejiang 310058, China
}

\section{Biomed Central

abnormal YAP/TAZ activation with human cancers [20-23]. However, upstream signals regulating the Hippo pathway and YAP/TAZ were obscure until the publication of several recent studies [24-28]. In this review we discuss the roles of YAP/TAZ as mediators of responses to mechanical stress, GPCR signaling and the Wnt signaling.

\section{Biochemical and biological functions of YAP/TAZ}

YAP was first cloned as a protein bound to non-receptor tyrosine kinase YES1 [29]. YAP mRNA is ubiquitously expressed in a wide range of tissues, except peripheral blood leukocytes [30]. There are two major splicing variants with one (YAP1) or two (YAP2) WW domains. The function of YAP remained enigmatic until it was shown to be a transcription co-activator [31]. A reporter assay demonstrated that the C-terminal region of YAP has strong transcriptional activation activity. However, it does not directly bind to DNA. Instead, it is brought to gene promoters through the interaction with transcription factors. First identified by affinity purification [32], TEAD family transcription factors are major partners of YAP in regulation of cell proliferation and organ size [17]. The importance of TEADs in the function of YAP was nicely demonstrated by a mouse model with knockin of a mutant YAP deficient in interaction with TEAD. Remarkably, the knock-in mice showed skin phenotypes 
closely resembled that of the YAP knock-out mice [33]. Furthermore, a heterozygous YAP-binding-deficient mutation of TEAD1 leads to a human genetic disease Sveinsson's chorioretinal atrophy $[17,34,35]$, which further illustrates the physiological importance of TEAD in YAP function. Through the centrally localized WW domains, YAP also interacts with other transcription factors such as RUNX1/2 and Smad1 [31,36], which may also contribute to YAPinduced gene expression and organ size control. The mechanism by which YAP activates transcription is still elusive. However, the Drosophila homolog of YAP, Yki, was shown to interact with GAGA factor (GAF), the Brahma complex, and the Mediator complex in cell nuclei to activate gene expression [37]. Interestingly, another report demonstrated that Scalloped (Sd), the Drosophila homolog of TEADs, function as a default transcription repressor by binding to Tgi [38]. When activated, Yki competes with Tgi for Sd binding and thus switches Sd from OFF to an ON state. Importantly, the function of Tgi is conserved in its mammalian homolog Vestigial-like 4 (Vgl4) [38].

YAP has an evolutionarily conserved function in organ size control. For instance, liver-specific overexpression of YAP in transgenic mice results in enlarged liver, which is reversible upon cessation of YAP overexpression $[14,39]$. However, sustained YAP overexpression eventually leads to the development of liver tumors [14]. Examination of clinical samples identified genomic amplification as well as elevated expression and nuclear localization of YAP in human cancers $[13,14,20,21,40]$. Further experiments on cultured cells supported the function of YAP in inducing cell transformation, loss of cell-contact-inhibition, and epithelial-mesenchymal transition (EMT) [13,41]. Thus current findings indicate an oncogenic role of YAP. YAP activation also promotes the expansion of tissue-specific progenitor cells such as in skin, chicken neural tube, and more controversially in intestine, which would be discussed below [39,42-44]. Furthermore, YAP promotes selfrenewal of mouse ES cells and represses differentiation [45]. Taken together, YAP has important functions in organ size control, tumorigenesis and tissue regeneration.

TAZ is a YAP paralog initially identified as a 14-3-3 binding protein [46]. In human and mouse, TAZ mRNA is expressed in all tissues except thymus and peripheral blood leukocytes, with the highest expression in kidney [46]. TAZ has approximately 50\% sequence identity and very similar topology with YAP. Clued by the transcription coactivator function of YAP, TAZ was also confirmed to have similar activity highly dependent on TEAD family transcription factors [16,46]. However, some target transcription factors are unique to TAZ, such as Smad2/3 and Pax3, which may contribute to the differential functions of TAZ to YAP [47,48]. TAZ also promotes cell proliferation, induces EMT, increases cell migration and invasion in vitro $[15,22]$, and is shown to be overexpressed in approximately $20 \%$ of breast cancer samples [22]. Interestingly, a recent study identified TAZ as a key factor sustaining self-renewal of breast cancer stem cells [23]. Despite the functional similarity of YAP and TAZ, they still possess some clearly distinct characters. For instance, YAP and TAZ knockout mice show different phenotypes: YAP knockout animals are embryonic lethal and show shortened body axis and defects in yolk sac vasculogenesis [49]. In contrast, TAZ knockout mice are viable and are characterized by renal cysts which lead to end stage kidney disease [50,51]. In addition, in many cases, the phenotype of YAP or TAZ knockdown were not compensated by the presence of the other $[17,22,47,52]$. Such differences may be explained by spatial and temporal regulation of YAP and TAZ activity or different downstream targets, which require further study.

\section{Mechanisms regulating YAP/TAZ activity}

YAP and TAZ regulate gene expression. However, the regulation on transcription of themselves is largely unknown. Recently it was reported that an Ets family transcription factor GABP directly promotes YAP transcription under inhibition by oxidative stress [53]. However, most of the known mechanisms regulating YAP/TAZ activity are on post-translational level. The physiological significance of YAP/TAZ was first revealed after the identification of Drosophila Yki as a key effector of the Hippo pathway [54]. Detailed biochemical analysis indicated that YAP is directly phosphorylated by Lats1/2 on five consensus HXRXXS motifs $[13-15,55,56]$. Phosphorylation of S127 in YAP promotes 14-3-3 binding, resulting in cytoplasmic sequestration and therefore inactivation of YAP $[13-15,55,56]$. Phosphorylation on YAP S381 primes subsequent phosphorylation by another kinase, possibly casein kinase 1 $(\mathrm{CK} 1 \delta / \varepsilon)$, thereby activates a phosphodegron degradation motif. Subsequently, the activated phosphodegron recruits the E3 ubiquitin ligase $\mathrm{SCF}^{\beta \text {-TRCP }}$, leading to polyubiquitination and degradation of YAP [57]. The importance of YAP as a downstream effector of the Hippo pathway was elegantly demonstrated in vivo by the reversal of Hippo pathway deficiency-induced oncogenic phenotypes by loss of one allele of YAP $[58,59]$. TAZ has four conserved Lats $1 / 2$ target motifs and is regulated by the Hippo pathway in a similar manner $[15,60]$.

Besides the canonical Hippo pathway, YAP/TAZ are also regulated by physical interactions with other proteins, especially cell junctional proteins. Through the WW domains, YAP could interact with angiomotin (AMOT) family proteins, which results in YAP localization to tight junction and YAP inhibition through phosphorylationdependent and -independent mechanisms [61-63]. YAP and TAZ also interact with another tight junction protein $\mathrm{ZO}-2$, which was reported to increase nuclear localization 
of YAP and tight-junction localization of TAZ, respectively $[64,65]$. Interestingly, a major adherens junction protein alpha-catenin, can also bind to and inhibit YAP by mediating its cell-cell junction and cytoplasmic localizations $[33,44]$. Another adherens junction protein PTPN14 has also been reported by several groups to be a negative regulator of YAP through the interaction with YAP WW domains [66-69]. In consistence with these finding on YAP/ TAZ regulation by junctional proteins, YAP/TAZ were found to be regulated by cell-cell contact. In tissue culture, high cell density induces YAP phosphorylation and cytoplasmic translocation [13] and disruption of cell-cell junctions results in the nuclear localization of YAP/TAZ [70]. In mouse blastocysts, YAP is nuclear in outer layer cells, and cytoplasmic in the inner blastocyst layer cells [71]. Taken together, it is clear that YAP/TAZ transcription coactivators might mediate upstream signals through both the Hippo pathway and their interactions with other proteins.

YAP/TAZ mediate cellular responses to mechanical stress Biomechanics is increasingly recognized as an important regulator of cell physiology and a key player in development and pathological abnormalities. For instance, many cancers such as breast cancer have elevated tissue stiffness due to altered ECM composition. Remarkably, softening of the tumor microenvironment slows tumor growth and progression [72]. It is also known that matrix stiffness is a determinant factor for lineage commitment of mesenchymal stem cells (MSCs). MSCs differentiate into adipocytes on soft matrix whereas osteoblasts on stiff matrix [73]. However, little was known about the molecular mechanisms transducing these conditions into the nucleus and resulting in physiological response. It was known that TAZ could promote osteogenesis and repress adipogenesis of MSCs possibly through activation of RUNX2dependent gene transcription and inhibition of PPAR $\gamma$ dependent gene expression [52]. This raises an interesting possibility of YAP/TAZ in mediating MSC differentiation in response to mechanical stress. This speculation was recently proved to be true [25]. When cells were grown on soft matrix or on micropatterned small islands, cells adopted a round shape and YAP/TAZ were mostly cytoplasmic. However, when cells were grown on stiff materials or large adhesive islands, they became nuclear and thus active. More importantly, the activity of YAP and TAZ determines the lineage commitment of MSCs in response to matrix stiffness. Cell adhesion and suspension are two conditions affect cell geometry in an analogous but more potent way than different matrix stiffness. It was found that YAP/TAZ subcellular localization is regulated by cell adhesion/suspension in a way similar to matrix stiffness [74]. Thus, YAP and TAZ are key nuclear effectors of mechanical stress.
Contraction of the actomyosin cytoskeleton plays a central role in generation and transducing mechanical forces in cells. Consistently, the regulation of YAP/TAZ localization by mechanical stress depends on F-actin and Rho family GTPases [25,74,75]. Disruption of F-actin or inhibition of Rho by specific inhibitors inactivates YAP. On the contrary, induced actin polymerization by overexpression of F-actin nucleator diaphanous correlates with activation of YAP/TAZ [76]. Regulation of the Drosophila Yki by F-actin has also been demonstrated in vivo [76]. Disruption of F-actin in vivo through several different genetic manipulations results in Yki activation and overgrowth of Drosophila tissue. Importantly, Yki was found to be required for the overgrowth.

The mechanism of YAP/TAZ regulation by cytoskeleton and mechanical stress is not completely understood. It was reported that knockdown of Lats1/2 is insufficient to rescue YAP/TAZ activity in cells cultured on soft matrix [25]. Nevertheless, in another report comparing cell attachment on stiff matrix or complete detachment, the Hippo pathway kinases Lats $1 / 2$ was found to be activated by cell detachment, also in a cytoskeleton-dependent manner [74]. And knockdown of Lats1/2 partially prevents mechanical stress-induced YAP phosphorylation and activation $[74,75]$. Thus it is possible that both Lats1/2-dependent and independent mechanisms are involved in the YAP/TAZ regulation by mechanical stress (Figure 1). However, the factors and molecular mechanisms behind each possibility are unclear and await further characterization.

The biological relevance of YAP/TAZ-mediated response to mechanical stress has recently been demonstrated in breast cancer. Breast tumor is featured by its higher stiffness compared with normal mammary tissue, which is due to excessive deposition of collagen by stromal cells. Interestingly, it was found that YAP is activated in cancerassociated fibroblasts (CAFs), and its function is required for matrix stiffing [24]. Matrix stiffing further enhances YAP activity and thus forms a positive feedback loop creating a cancerous microenvironment. It was proposed that YAP conditioned the tumor microenvironment by stiffing the matrix to promote cancer cell invasion, although the role of YAP-induced secreted factors in this process was not formally excluded. Such activity in CAFs is observed only for YAP but not for TAZ. Nevertheless, only TAZ but not YAP was found to be associated with CSC maintenance and tumor progression in breast cancer [23]. Whether TAZ in epithelial cancer cells is activated by YAP-induced matrix stiffing awaits further confirmation. However, there seems to be an interesting cell-type specificity and job division between YAP and TAZ in the promotion of breast cancer.

\section{YAP/TAZ are effectors of GPCR signaling}

Soluble molecules such as growth factors, morphogens, cytokines, and hormones serve as key factors initiating 


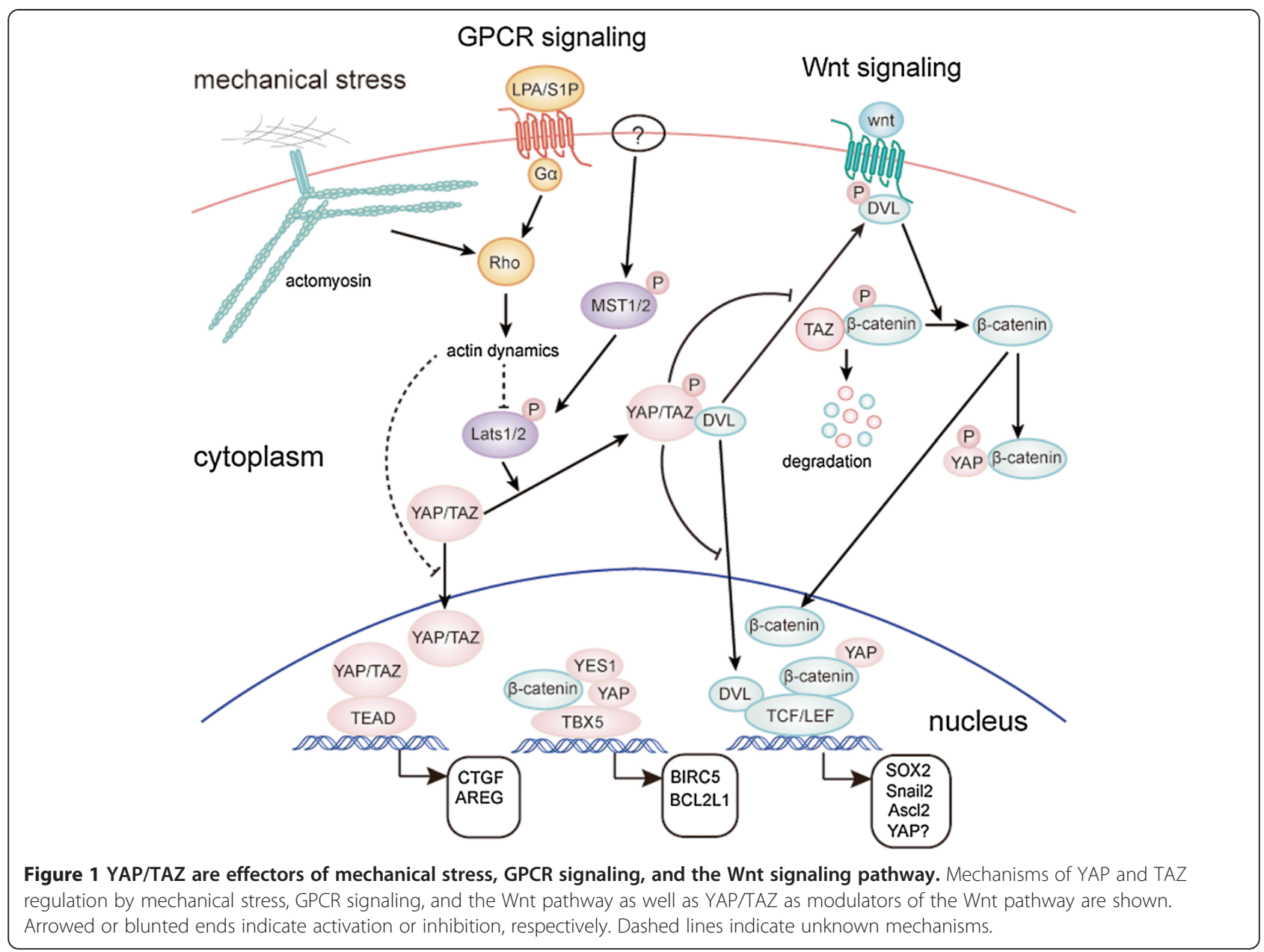

intracellular signaling pathways through association with and activation of their cognate receptors. In fact, most known signaling pathways involved in growth control in development and cancer are triggered by such extracellular cues. Despite the discovery of mechanical stress as a special upstream signal for YAP/TAZ, a soluble molecule as stimulant of the Hippo pathway was elusive. It was even unclear whether such a molecule exists. Nevertheless, two recent reports identified lysophosphatidic acid (LPA) and sphingosine-1-phosphophate (S1P), two related phospholipids in serum as potent activator of YAP/TAZ [26,27] (Figure 1). Following the initial observation of serum as a strong stimulant of YAP dephosphorylation and nuclear localization, both groups identified LPA and S1P as the active ingredients through a series of elegant biochemical analysis. Further experiments demonstrated that LPA and S1P activate YAP/ TAZ by binding to their respective G-protein-coupled receptors (GPCRs) on cell surface and activation of the downstream heterotrimeric G proteins. Rho GTPase and remodeling of F-actin are known effectors of GPCR signaling and are also required for YAP/TAZ regulation by
LPA and S1P. Thus the regulation of YAP/TAZ by mechanical stress and soluble factors converge on actin cytoskeleton remodeling. Activation of LPA and S1P receptors leads to inhibition of Lats1/2 kinase activity and knockdown of Lats1/2 partially blocks YAP phosphorylation induced by serum deprivation [26,27]. Thus Lats1/ 2 is at least partly responsible for YAP/TAZ regulation by LPA and S1P, although current evidence does not exclude the possibility of other mechanisms being involved in the process. However, Mst1/2 kinase activity is not regulated by these signals and ablation of Mst1/2 does not impair YAP/TAZ response to GPCR signaling $[26,27]$. This suggests that other molecules might be involved in regulation of the Hippo pathway kinases Lats $1 / 2$ in response to GPCR signaling.

It was later reported that thrombin, which activates protease-activated receptors, another GPCR, also stimulates YAP and TAZ activity [77]. In fact, it was found that YAP/TAZ is robustly regulated by many GPCRs and their cognate ligands [27]. In addition, YAP/TAZ activity could be either activated or inhibited depending on the $\mathrm{G}$ proteins coupled to the receptors. For example, 
activation of $\mathrm{G \alpha}_{12 / 13}, \mathrm{G} \alpha_{\mathrm{q} / 11}$, or $\mathrm{G \alpha}_{\mathrm{i} / \mathrm{o}}$ induces YAP/TAZ activity, whereas activation of $\mathrm{G \alpha}_{\mathrm{s}}$ represses YAP/TAZ activity [27]. Therefore, YAP/TAZ seems to be a common target of GPCR signaling. It would then be interesting to determine whether these regulations on YAP/TAZ are all executed through a similar mechanism. And if not, what would be the individual mechanisms and for what kind of logic do these different signals converge on the regulation of YAP/TAZ.

GPCRs are the largest family of cell surface receptors mediating responses to a wide range of physiological signals and importantly, medicines [78]. Abnormal GPCR signaling is also involved in cancer development in many ways. Elevated expression of GCPRs such as PAR1 was found in high-grade breast cancers [79]. Furthermore, activating mutations of GPCRs have been found in several types of cancers such as melanomas and thyroid carcinomas [80,81]. In addition, activating mutations of $\mathrm{G} \alpha$ proteins have also been found in cancers, which is best exemplified by the remarkable $\mathrm{G \alpha}_{\mathrm{q} / 11}$ activating mutation rate of more than $80 \%$ in uveal melanomas $[82,83]$. It was reported that transgenic expression of LPA receptor 2 in mouse mammary glands induces activation of YAP/TAZ and massive overgrowth $[27,84]$. Thus, activation of YAP/TAZ might be involved in cancer induced by aberrant GPCR signaling. Such a possibility would need to be validated by experiments. Besides the pathological role of the GPCR-YAP/TAZ axis, it is also important to determine the role of this mechanism in organ size control in development and regeneration. Providing the large pool of circulating GPCR ligands including LPA and S1P, it would be important to identify the key player in organ size control in vivo.

\section{YAP/TAZ as effectors and modulators of Wnt signaling}

Wnt is an important morphogen in development. In the canonical Wnt signaling pathway stimulation of Wnt receptors on cell surface results in disassembly of the $\beta$-catenin destruction complex. As a result, $\beta$-catenin accumulates in cell nuclei to stimulate expression of Wnt target genes [85]. In this way, $\beta$-catenin is a widely accepted effector of Wnt signaling pathway. Interestingly, a recent report discovered an unexpected role of TAZ as an effector of the Wnt pathway [28]. It was found that Wnt stimulation leads to stabilization of $\beta$-catenin as well as TAZ, but not YAP. TAZ physically interact with $\beta$-catenin and knockdown of $\beta$-catenin increases TAZ protein level and activity. It was proposed that $\beta$-catenin directs TAZ for co-degradation through $\mathrm{SCF}^{\beta-\mathrm{TRCP}}$ mediated ubiquitination (Figure 1). Previously studies demonstrated that TAZ stability is regulated by phosphorylation of a C-terminal phospho-degron by the Hippo pathway and phosphorylation of an N-terminal degron by GSK3 $[60,86]$. Relationship between the three mechanisms has not been established yet. Noteworthy, it was also reported that Wnt/ $\beta$-catenin promoted YAP protein level by activating YAP transcription [87]. Strikingly, gene expression profiling of mammary epithelial cells with knockdown of $\beta$-catenin or TAZ revealed that $74 \%$ of $\beta$-catenin target genes are also dependent on TAZ [28]. Such observations would suggest TAZ as a fundamental effector of the Wnt signaling pathway. However, the physiological or pathological relevance of this hypothesis is yet to be validated. Obviously, activation or inhibition of the Wnt pathway and the Hippo pathway results in very different phenotypes in animals suggesting differential roles in development. Furthermore, it was unclear whether TAZ is activated and plays a functional role in colon cancers, where abnormal activation of $\beta$-catenin plays a key role in tumorigenesis. In fact, another report published back-to-back with the above study identified YAP, but not TAZ as an essential survival factor for $\beta$-catenin-driven cancer cell lines [88]. In this study, 85 cancer cell lines were divided into $\beta$-catenin active and inactive groups based on TCF4 reporter activity. Further RNAi screen identified YAP as an essential gene for survival and anchorage-independent growth of $\beta$-catenin active cancer cell lines. However, suppression of TAZ expression did not affect the proliferation of these cell lines. Surprisingly, further experiments indicated that YAP supported the survival of $\beta$-catenin active cancer cells by partner with transcription factor TBX5 under the help of tyrosine kinase YES1. This transcriptional complex stimulates expression of genes such as BCL2L1 and BIRC5 to support cancer cell survival. However, the involvement of major YAP/TAZ target transcription factors, the TEAD family proteins, has not been excluded by experiments. Although TCF4 is the classical transcription factor partner of $\beta$-catenin, the report showed that YAP did not activate $\beta$-catenin on the TCF4 reporter. Nevertheless, in some other context such as mouse intestine and cardiomyocytes, activation of YAP due to inhibition of the Hippo pathway correlates with activation of $\beta$-catenin/TCF4 target genes $[58,89]$. Furthermore, YAP co-occupy gene promoters with $\beta$-catenin [89]. Therefore, the functional transcription factor partners of the YAP- $\beta$-catenin complex are not completely understood and could be context-dependent. Nevertheless, the above studies suggest a nuclear role of YAP/TAZ in modulating gene expression regulation and biological effects of the Wnt signaling pathway (Figure 1).

However, YAP/TAZ may also modulate the Wnt pathway through cytoplasmic mechanisms (Figure 1). In cultured cells, it was found that overexpression of YAP/ TAZ inhibits $\beta$-catenin/TCF4 reporter activity and knockdown of YAP/TAZ activates it $[90,91]$. At the first look this would be contradictory to the nuclear function of YAP/TAZ in promoting beta-catenin activity. However, it was then demonstrated that cytoplasmic YAP and TAZ are the main force in inhibiting $\beta$-catenin. 
Cytoplasmic YAP may directly sequester $\beta$-catenin in the cytoplasm or cytoplasmic TAZ may sequester DVL2 impeding its activity to promote $\beta$-catenin accumulation in response to Wnt stimulation $[90,91]$. So that nuclear and cytoplasmic YAP/TAZ may have opposite roles in regulating $\beta$-catenin activity. The Hippo pathway is the best known mechanism for promoting cytoplasmic localization of YAP/TAZ. Thus the Hippo pathway may inhibit Wnt signaling through two distinct mechanisms, by repressing YAP/TAZ/ $\beta$-catenin activity in cell nuclei and by promoting $\beta$-catenin cytoplasmic retention and possibly degradation.

Wnt pathway plays a key role in intestinal stem cell selfrenewal and intestinal regeneration [85]. The function of YAP/TAZ in intestinal regeneration was also examined. Consistent with the role of YAP in promoting $\beta$-catenin activity in the nucleus and the growth promoting activity of itself, it was found that inactivation of YAP severely impairs dextran sodium sulfate (DSS)-induced intestinal regeneration, although YAP activity seems dispensable during development and normal homeostasis [18]. Noteworthy, the absence of phenotypes in YAP knockout intestines could be due to compensation by TAZ. During regeneration, YAP protein level was found to be elevated. Similarly, inactivation of Mst1 and Mst2 in mouse intestine also increases the protein level of YAP [58]. It was unclear whether the change of YAP protein level is related to Wnt signaling. However, further another group demonstrated that in irradiation-induced intestine regeneration model, loss of YAP leads to overexpansion of intestinal stem cells and development of microadenomas, which correlates with hyperactive Wnt signaling [19]. This finding would support an inhibitory role of YAP on Wnt signaling during regeneration although the underlying reason for differential YAP functions in the two intestine regeneration models is unknown. More surprisingly, although it was previously demonstrated that transgenic expression of an active YAP-S127A mutant strongly expands intestinal progenitors [39], tissue-specific expression of wild-type YAP leads to a progressive degeneration phenotype associated with loss of crypts and hypoactive Wnt signaling [19]. This would indicate that YAP, even the transgenicly expressed YAP is under inhibition by the Hippo pathway in intestinal epithelium, so that the Hippo pathway hypo-responsive S127A YAP mutant has different function from the wildtype protein. Furthermore, other than simply inactive, the extra abundant presumably cytoplasmic wild-type YAP protein plays some roles other than the nuclear YAP protein. The activity is possible as proposed to be the inhibition of $\beta$-catenin activity by restricting nuclear localization of DVL. It is worth noting that the intestinal phenotypes and altered Wnt signaling activity observed in wild-type YAP transgenic model and in YAP knockout model under irradiation-induced regeneration condition may be alternatively explained by loss or gain of Paneth cells due to the activity of YAP on cell differentiation [92]. Paneth cells serve as an important part of the stem cell niche and a major source of the Wnt ligand. Thus clarify the functions of YAP/TAZ in intestinal stem cells and their niche would be a crucial future direction. We summarized the complex phenotypes of intestinal crypt cells in reported mouse models concerning the cross-talk between the Hippo and Wnt pathways (Figure 2). Similar to the situation in intestinal regeneration, there are also contradictory reports on the correlation between YAP and colon cancer. YAP was reported to be both elevated in cancer and associated with a better prognosis $[19,21,58]$. A better understanding of the role of YAP in this disease would need a more quantitative assay for YAP level and localization and more high quality patient samples with disease progression data.

\section{Conclusions}

YAP and TAZ transcription coactivators play key roles in organ size control, regeneration, and cancer development.

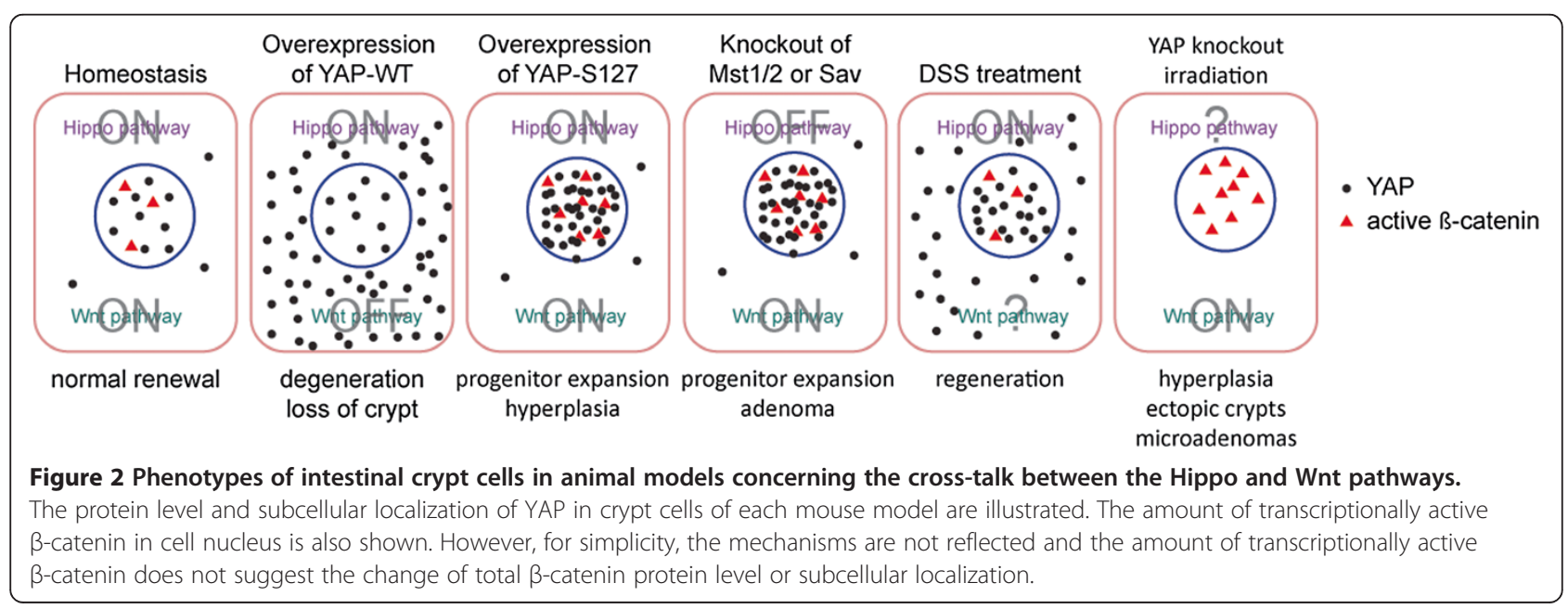


Despite elucidation of their regulation by the Hippo pathway mediated phosphorylation and other mechanisms, the upstream physiological signals controlling YAP/TAZ activity has been elusive for a long time. Nevertheless, recent discoveries of YAP/TAZ as mediators of mechanical stress, GPCR signaling, and Wnt signaling open up the window to understand YAP/TAZ regulation under a complex physiological context in vivo with both physical and chemical properties. However, it is important to realize that in all three cases, key molecular mechanisms are still missing or are complicated by contradictory reports. For example, it is unclear how F-actin cytoskeleton regulates Lats1/2 kinase activity, which is important for YAP/TAZ regulation by both mechanical stress and GPCR signaling. Furthermore, identify of the possibly existing Lats1/2-independent mechanism of YAP/TAZ regulation by F-actin remodeling is yet to be uncovered. Moreover, the nuclear and cytoplasmic roles of YAP/TAZ in regulation of $\beta$-catenin activity need to be clarified and the mechanisms await further validation. In addition, the specificity and possibly differential roles of YAP and TAZ in mediating the above signals and in tissue regeneration and cancer also require more precise assessments. It is also interesting to understand how YAP/TAZ may serve to integrate different signals to mediate a proper response to the dynamic in vivo environment. For example, both mechanical stress and GPCR signaling input into regulation of Rho activity and thus affects YAP/TAZ activity. Therefore, it is interesting to know whether Rho serve as a checkpoint for both the physiological environment and the availability of chemical GPCR ligands to decide on cell proliferation and differentiation. Despite the existence of so many questions to be answered, the YAP/TAZ transcription coactivators are undoubtedly important mediators of physiological signals in regulation of organ size control, regeneration and tumorigenesis.

\section{Competing interests}

The authors declare that they have no competing interests.

\section{Authors' contributions}

XG prepared the illustrations. BZ conceived the manuscript. XG and BZ drafted the manuscript. Both authors read and approved the final manuscript.

\section{Acknowledgements}

Research in the lab of Bin Zhao is supported by grants from the Natural Science Foundation of Zhejiang (LR12C07001), State Key Development Program for Basic Research of China (2013CB945303), National Natural Science Foundation of China (31271508), and the Thousand Young Talents Plan of China.

Received: 14 June 2013 Accepted: 30 June 2013

Published: 28 August 2013

\section{References}

1. Zhao B, Li L, Lei Q, Guan KL: The Hippo-YAP pathway in organ size control and tumorigenesis: an updated version. Genes Dev 2010 24(9):862-874
2. Yu F-X, Guan K-L: The Hippo pathway: regulators and regulations. Genes Dev 2013, 27(4):355-371.

3. $\mathrm{X} u$ T, Wang W, Zhang S, Stewart RA, Yu W: Identifying tumor suppressors in genetic mosaics: the Drosophila lats gene encodes a putative protein kinase. Development 1995, 121(4):1053-1063.

4. Justice RW, Zilian O, Woods DF, Noll M, Bryant PJ: The Drosophila tumor suppressor gene warts encodes a homolog of human myotonic dystrophy kinase and is required for the control of cell shape and proliferation. Genes Dev 1995, 9(5):534-546.

5. Kango-Singh $M$, Nolo R, Tao C, Verstreken P, Hiesinger PR, Bellen $H J$, Halder G: Shar-pei mediates cell proliferation arrest during imaginal disc growth in Drosophila. Development 2002, 129(24):5719-5730.

6. Tapon N, Harvey KF, Bell DW, Wahrer DC, Schiripo TA, Haber DA, Hariharan IK: Salvador Promotes both cell cycle exit and apoptosis in Drosophila and is mutated in human cancer cell lines. Cell 2002, 110(4):467-478.

7. Harvey KF, Pfleger CM, Hariharan IK: The Drosophila Mst ortholog, hippo, restricts growth and cell proliferation and promotes apoptosis. Cell 2003, 114(4):457-467.

8. Wu S, Huang J, Dong J, Pan D: Hippo encodes a Ste-20 family protein kinase that restricts cell proliferation and promotes apoptosis in conjunction with salvador and warts. Cell 2003, 114(4):445-456.

9. Udan RS, Kango-Singh M, Nolo R, Tao C, Halder G: Hippo promotes proliferation arrest and apoptosis in the Salvador/Warts pathway. Nat Cell Biol 2003, 5(10):914-920.

10. Pantalacci S, Tapon N, Leopold P: The Salvador partner Hippo promotes apoptosis and cell-cycle exit in Drosophila. Nat Cell Biol 2003, $5(10): 921-927$.

11. Jia J, Zhang W, Wang B, Trinko R, Jiang J: The Drosophila Ste20 family kinase dMST functions as a tumor suppressor by restricting cell proliferation and promoting apoptosis. Genes Dev 2003, 17(20):2514-2519.

12. Lai ZC, Wei X, Shimizu T, Ramos E, Rohrbaugh M, Nikolaidis N, Ho LL, Li Y: Control of cell proliferation and apoptosis by mob as tumor suppressor, mats. Cell 2005, 120(5):675-685.

13. Zhao B, Wei X, Li W, Udan RS, Yang Q, Kim J, Xie J, Ikenoue T, Yu J, Li L, et al: Inactivation of YAP oncoprotein by the Hippo pathway is involved in cell contact inhibition and tissue growth control. Genes Dev 2007, 21(21):2747-2761.

14. Dong J, Feldmann G, Huang J, Wu S, Zhang N, Comerford SA, Gayyed MF, Anders RA, Maitra A, Pan D: Elucidation of a universal size-control mechanism in Drosophila and mammals. Cell 2007, 130(6):1120-1133.

15. Lei QY, Zhang H, Zhao B, Zha ZY, Bai F, Pei XH, Zhao S, Xiong Y, Guan KL: TAZ promotes cell proliferation and epithelial-mesenchymal transition and is inhibited by the hippo pathway. Mol Cell Biol 2008, 28(7):2426-2436

16. Zhang H, Liu CY, Zha ZY, Zhao B, Yao J, Zhao S, Xiong Y, Lei QY, Guan KL: TEAD transcription factors mediate the function of TAZ in cell growth and epithelial-mesenchymal transition. J Biol Chem 2009, 284(20):13355-13362.

17. Zhao B, Ye X, Yu J, Li L, Li W, Li S, Lin JD, Wang CY, Chinnaiyan AM, Lai ZC et al: TEAD mediates YAP-dependent gene induction and growth control. Genes Dev 2008, 22(14):1962-1971.

18. Cai J, Zhang N, Zheng Y, de Wilde RF, Maitra A, Pan D: The Hippo signaling pathway restricts the oncogenic potential of an intestinal regeneration program. Genes Dev 2010, 24(21):2383-2388.

19. Barry ER, Morikawa T, Butler BL, Shrestha K, de La Rosa R, Yan KS, Fuchs CS, Magness ST, Smits R, Ogino S: Restriction of intestinal stem cell expansion and the regenerative response by YAP. Nature 2012, 493(7430):106-110.

20. Zender L, Spector MS, Xue W, Flemming P, Cordon-Cardo C, Silke J, Fan ST, Luk JM, Wigler M, Hannon GJ, et al: Identification and validation of oncogenes in liver cancer using an integrative oncogenomic approach. Cell 2006, 125(7):1253-1267.

21. Steinhardt AA, Gayyed MF, Klein AP, Dong J, Maitra A, Pan D, Montgomery EA, Anders RA: Expression of Yes-associated protein in common solid tumors. Hum Pathol 2008, 39(11):1582-1589.

22. Chan SW, Lim CJ, Guo K, Ng CP, Lee I, Hunziker W, Zeng Q, Hong W: A role for TAZ in migration, invasion, and tumorigenesis of breast cancer cells. Cancer Res 2008, 68(8):2592-2598.

23. Cordenonsi M, Zanconato F, Azzolin L, Forcato M, Rosato A, Frasson C, Inui M, Montagner M, Parenti AR, Poletti A, et al: The Hippo transducer TAZ confers cancer stem cell-related traits on breast cancer cells. Cell 2011 147(4):759-772. 
24. Calvo F, Ege N, Grande-Garcia A, Hooper S, Jenkins RP, Chaudhry SI, Harrington K, Williamson P, Moeendarbary E, Charras G: Mechanotransduction and YAP-dependent matrix remodelling is required for the generation and maintenance of cancer-associated fibroblasts. Nat Cell Biol 2013, 15(6):637-646.

25. Dupont S, Morsut L, Aragona M, Enzo E, Giulitti S, Cordenonsi M, Zanconato F, Le Digabel J, Forcato M, Bicciato S, et al: Role of YAP/TAZ in mechanotransduction. Nature 2011, 474(7350):179-183.

26. Miller E, Yang J, DeRan M, Wu C, Su Al, Bonamy G, Liu J, Peters EC, Wu X: Identification of serum-derived sphingosine-1-phosphate as a small molecule regulator of YAP. Chem Biol 2012, 19(8):955-962.

27. Yu FX, Zhao B, Panupinthu N, Jewell JL, Lian I, Wang LH, Zhao J, Yuan H, Tumaneng K, Li H, et al: Regulation of the Hippo-YAP pathway by G-protein-coupled receptor signaling. Cell 2012, 150(4):780-791.

28. Azzolin L, Zanconato F, Bresolin S, Forcato M, Basso G, Bicciato S, Cordenonsi M, Piccolo S: Role of TAZ as mediator of Wnt signaling. Cell 2012, 151(7):1443-1456.

29. Sudol M: Yes-associated protein (YAP65) is a proline-rich phosphoprotein that binds to the $\mathrm{SH} 3$ domain of the Yes proto-oncogene product. Oncogene 1994, 9(8):2145-2152.

30. Komuro A, Nagai M, Navin NE, Sudol M: WW domain-containing protein YAP associates with ErbB-4 and acts as a co-transcriptional activator for the carboxyl-terminal fragment of ErbB-4 that translocates to the nucleus. J Biol Chem 2003, 278(35):33334-33341.

31. Yagi $R$, Chen LF, Shigesada $K$, Murakami $Y$, Ito $Y$ : A WW domain-containing yes-associated protein (YAP) is a novel transcriptional co-activator. EMBO J 1999, 18(9):2551-2562.

32. Vassilev A, Kaneko KJ, Shu H, Zhao Y, DePamphilis ML: TEAD/TEF transcription factors utilize the activation domain of YAP65, a Src/Yesassociated protein localized in the cytoplasm. Genes Dev 2001, 15(10):1229-1241

33. Schlegelmilch K, Mohseni M, Kirak O, Pruszak J, Rodriguez JR, Zhou D, Kreger BT, Vasioukhin V, Avruch J, Brummelkamp TR, et al: Yap1 acts downstream of alpha-catenin to control epidermal proliferation. Cell 2011, 144(5):782-795.

34. Fossdal R, Jonasson F, Kristjansdottir GT, Kong A, Stefansson H, Gosh S Gulcher JR, Stefansson K: A novel TEAD1 mutation is the causative allele in Sveinsson's chorioretinal atrophy (helicoid peripapillary chorioretinal degeneration). Hum Mol Genet 2004, 13(9):975-981.

35. Kitagawa M: A Sveinsson's chorioretinal atrophy-associated missense mutation in mouse Tead1 affects its interaction with the co-factors YAP and TAZ. Biochem Biophys Res Commun 2007, 361(4):1022-1026.

36. Alarcon C, Zaromytidou Al, Xi Q, Gao S, Yu J, Fujisawa S, Barlas A, Miller AN Manova-Todorova K, Macias MJ, et al: Nuclear CDKs drive Smad transcriptional activation and turnover in BMP and TGF-beta pathways. Cell 2009, 139(4):757-769.

37. Oh H, Slattery M, Ma L, Crofts A, White KP, Mann RS, Irvine KD: Genomewide association of Yorkie with chromatin and chromatin-remodeling complexes. Cell Rep 2013, 3(2):309-318.

38. Koontz LM, Liu-Chittenden Y, Yin F, Zheng Y, Yu J, Huang B, Chen Q, Wu S, Pan $D$ : The hippo effector yorkie controls normal tissue growth by antagonizing scalloped-mediated default repression. Dev Cell 2013, 25(4):388-401.

39. Camargo FD, Gokhale S, Johnnidis JB, Fu D, Bell GW, Jaenisch R, Brummelkamp TR: YAP1 increases organ size and expands undifferentiated progenitor cells. Curr Biol 2007, 17(23):2054-2060.

40. Hall CA, Wang R, Miao J, Oliva E, Shen X, Wheeler T, Hilsenbeck SG, Orsulic S, Goode S: Hippo pathway effector Yap is an ovarian cancer oncogene. Cancer Res 2010, 70(21):8517-8525.

41. Overholtzer M, Zhang J, Smolen GA, Muir B, Li W, Sgroi DC, Deng CX, Brugge JS, Haber DA: Transforming properties of YAP, a candidate oncogene on the chromosome 11q22 amplicon. Proc Natl Acad Sci U S A 2006, 103(33):12405-12410.

42. Cao X, Pfaff SL, Gage FH: YAP regulates neural progenitor cell number via the TEA domain transcription factor. Genes Dev 2008, 22(23):3320-3334.

43. Zhang $H$, Pasolli HA, Fuchs E: Yes-associated protein (YAP) transcriptional coactivator functions in balancing growth and differentiation in skin. Proc Natl Acad Sci U S A 2011, 108(6):2270-2275.

44. Silvis MR, Kreger BT, Lien WH, Klezovitch O, Rudakova GM, Camargo FD, Lantz DM, Seykora JT, Vasioukhin V: a-Catenin Is a Tumor Suppressor That Controls Cell Accumulation by Regulating the Localization and Activity of the Transcriptional Coactivator Yap1. Sci Signal 2011, 4(174):ra33.
45. Lian I, Kim J, Okazawa H, Zhao J, Zhao B, Yu J, Chinnaiyan A, Israel MA, Goldstein $L S$, Abujarour $R$, et al: The role of YAP transcription coactivator in regulating stem cell self-renewal and differentiation. Genes Dev 2010, 24(11):1106-1118.

46. Kanai F, Marignani PA, Sarbassova D, Yagi R, Hall RA, Donowitz M, Hisaminato A, Fujiwara T, Ito Y, Cantley LC, et al: TAZ: a novel transcriptional co-activator regulated by interactions with $14-3-3$ and PDZ domain proteins. EMBO J 2000, 19(24):6778-6791.

47. Varelas $X$, Sakuma R, Samavarchi-Tehrani P, Peerani R, Rao BM, Dembowy J, Yaffe MB, Zandstra PW, Wrana JL: TAZ controls Smad nucleocytoplasmic shuttling and regulates human embryonic stem-cell self-renewal. Nat Cell Biol 2008, 10(7):837-848.

48. Murakami M, Tominaga J, Makita R, Uchijima Y, Kurihara Y, Nakagawa O, Asano T, Kurihara H: Transcriptional activity of Pax3 is co-activated by TAZ. Biochem Biophys Res Commun 2006, 339(2):533-539.

49. Morin-Kensicki EM, Boone BN, Howell M, Stonebraker JR, Teed J, Alb JG, Magnuson TR, O'Neal W, Milgram SL: Defects in yolk sac vasculogenesis, chorioallantoic fusion, and embryonic axis elongation in mice with targeted disruption of Yap65. Mol Cell Biol 2006, 26(1):77-87.

50. Makita R, Uchijima Y, Nishiyama K, Amano T, Chen Q, Takeuchi T, Mitani A, Nagase T, Yatomi $Y$, Aburatani $H$, et al: Multiple renal cysts, urinary concentration defects, and pulmonary emphysematous changes in mice lacking TAZ. Am J Physiol Renal Physiol 2008, 294(3):F542-F553.

51. Hossain Z, Ali SM, Ko HL, Xu J, Ng CP, Guo K, Qi Z, Ponniah S, Hong W Hunziker W: Glomerulocystic kidney disease in mice with a targeted inactivation of Wwtr1. Proc Natl Acad Sci U S A 2007, 104(5):1631-1636.

52. Hong JH, Hwang ES, McManus MT, Amsterdam A, Tian Y, Kalmukova R, Mueller E, Benjamin T, Spiegelman BM, Sharp PA, et al: TAZ, a transcriptional modulator of mesenchymal stem cell differentiation. Science 2005, 309(5737):1074-1078.

53. Wu H, Xiao Y, Zhang S, Ji S, Wei L, Fan F, Geng J, Tian J, Sun X, Qin F, et al: The Ets Transcription Factor GABP Is a Component of the Hippo Pathway Essential for Growth and Antioxidant Defense. Cell Rep 2013, 3(5):1663-1677.

54. Huang J, Wu S, Barrera J, Matthews K, Pan D: The Hippo signaling pathway coordinately regulates cell proliferation and apoptosis by inactivating Yorkie, the Drosophila Homolog of YAP. Cell 2005, 122(3):421-434.

55. Hao Y, Chun A, Cheung K, Rashidi B, Yang X: Tumor suppressor LATS1 is a negative regulator of oncogene YAP. J Biol Chem 2008, 283(9):5496-5509.

56. Oka T, Mazack V, Sudol M: Mst2 and Lats kinases regulate apoptotic function of Yes kinase-associated protein (YAP). J Biol Chem 2008, 283(41):27534-27546.

57. Zhao B, Li L, Tumaneng K, Wang CY, Guan KL: A coordinated phosphorylation by Lats and CK1 regulates YAP stability through SCF (beta-TRCP). Genes Dev 2010, 24(1):72-85.

58. Zhou D, Zhang Y, Wu H, Barry E, Yin Y, Lawrence E, Dawson D, Willis JE, Markowitz SD, Camargo FD, et al: Mst1 and Mst2 protein kinases restrain intestinal stem cell proliferation and colonic tumorigenesis by inhibition of Yes-associated protein (Yap) overabundance. Proc Natl Acad Sci U S A 2011, 108(49):E1312-E1320.

59. Zhang N, Bai H, David KK, Dong J, Zheng Y, Cai J, Giovannini M, Liu P, Anders RA, Pan D: The Merlin/NF2 tumor suppressor functions through the YAP oncoprotein to regulate tissue homeostasis in mammals. Dev Cell 2010, 19(1):27-38.

60. Liu CY, Zha ZY, Zhou X, Zhang H, Huang W, Zhao D, Li T, Chan SW, Lim CJ, Hong W, et al: The hippo tumor pathway promotes TAZ degradation by phosphorylating a phosphodegron and recruiting the SCF\{beta\}-TrCP E3 ligase. J Biol Chem 2010, 285(48):37159-37169.

61. Chan SW, Lim CJ, Chong YF, Venkatesan Pobbati A, Huang C, Hong W: Hippo pathway-independent restriction of TAZ and YAP by angiomotin. J Biol Chem 2011, 286(9):7018-7026.

62. Wang W, Huang J, Chen J: Angiomotin-like proteins associate with and negatively regulate YAP1. J Biol Chem 2011, 286(6):4364-4370.

63. Zhao B, Li L, Lu Q, Wang LH, Liu CY, Lei Q, Guan KL: Angiomotin is a novel Hippo pathway component that inhibits YAP oncoprotein. Genes Dev 2011, 25(1):51-63.

64. Oka T, Remue E, Meerschaert K, Vanloo B, Boucherie C, Gfeller D, Bader GD, Sidhu SS, Vandekerckhove J, Gettemans J, et al: Functional complexes between YAP2 and ZO-2 are PDZ domain-dependent, and regulate YAP2 nuclear localization and signalling. Biochem J 2010, 432(3):461-472.

65. Remue E, Meerschaert K, Oka T, Boucherie C, Vandekerckhove J, Sudol M, Gettemans J: TAZ interacts with zonula occludens -1 and -2 proteins in a PDZ-1 dependent manner. FEBS Lett 2010, 584(19):4175-4180. 
66. Michaloglou C, Lehmann W, Martin T, Delaunay C, Hueber A, Barys L, Niu H, Billy E, Wartmann M, Ito $M$, et al: The tyrosine phosphatase PTPN14 is a negative regulator of YAP activity. PLoS One 2013, 8(4):e61916.

67. Liu X, Yang N, Figel SA, Wilson KE, Morrison CD, Gelman IH, Zhang J: PTPN14 interacts with and negatively regulates the oncogenic function of YAP. Oncogene 2013, 32(10):1266-1273.

68. Huang JM, Nagatomo I, Suzuki E, Mizuno T, Kumagai T, Berezov A, Zhang H, Karlan B, Greene MI, Wang Q: YAP modifies cancer cell sensitivity to EGFR and survivin inhibitors and is negatively regulated by the non-receptor type protein tyrosine phosphatase 14. Oncogene 2013, 32(17):2220-2229.

69. Wang W, Huang J, Wang X, Yuan J, Li X, Feng L, Park JI, Chen J: PTPN14 is required for the density-dependent control of YAP1. Genes Dev 2012, 26(17):1959-1971.

70. Varelas $X$, Samavarchi-Tehrani P, Narimatsu M, Weiss A, Cockburn K, Larsen BG, Rossant J, Wrana JL: The crumbs complex couples cell density sensing to Hippo-dependent control of the TGF-beta-SMAD pathway. Dev Cell 2010, 19(6):831-844

71. Nishioka N, Inoue K, Adachi K, Kiyonari H, Ota M, Ralston A, Yabuta N, Hirahara S, Stephenson RO, Ogonuki N, et al: The Hippo signaling pathway components Lats and Yap pattern Tead4 activity to distinguish mouse trophectoderm from inner cell mass. Dev Cell 2009, 16(3):398-410.

72. Levental KR, Yu H, Kass L, Lakins JN, Egeblad M, Erler JT, Fong SF, Csiszar K, Giaccia A, Weninger W, et al: Matrix crosslinking forces tumor progression by enhancing integrin signaling. Cell 2009, 139(5):891-906.

73. Engler AJ, Sen S, Sweeney HL, Discher DE: Matrix elasticity directs stem cell lineage specification. Cell 2006, 126(4):677-689.

74. Zhao B, Li L, Wang L, Wang CY, Yu J, Guan KL: Cell detachment activates the Hippo pathway via cytoskeleton reorganization to induce anoikis. Genes Dev 2012, 26(1):54-68.

75. Wada K, Itoga K, Okano T, Yonemura S, Sasaki H: Hippo pathway regulation by cell morphology and stress fibers. Development 2011, 138(18):3907-3914

76. Sansores-Garcia L, Bossuyt W, Wada K, Yonemura S, Tao C, Sasaki H, Halder $\mathrm{G}$ : Modulating F-actin organization induces organ growth by affecting the Hippo pathway. EMBO J 2011, 30(12):2325-2335.

77. Mo J-S, Yu F-X, Gong R, Brown JH, Guan K-L: Regulation of the Hippo-YAP pathway by protease-activated receptors (PARs). Genes Dev 2012, 26(19):2138-2143.

78. Lappano R, Maggiolini M: G protein-coupled receptors: novel targets for drug discovery in cancer. Nat Rev Drug Discov 2011, 10(1):47-60.

79. Hernandez NA, Correa E, Avila EP, Vela TA, Perez VM: PAR1 is selectively over expressed in high grade breast cancer patients: a cohort study. J Transl Med 2009, 7:47.

80. Paschke $\mathrm{R}$, Ludgate $\mathrm{M}$ : The thyrotropin receptor in thyroid diseases. N Engl J Med 1997, 337(23):1675-1681.

81. Prickett TD, Wei X, Cardenas-Navia I, Teer JK, Lin JC, Walia V, Gartner J, Jiang J, Cherukuri PF, Molinolo A, et al: Exon capture analysis of $\mathrm{G}$ proteincoupled receptors identifies activating mutations in GRM3 in melanoma. Nat Genet 2011, 43(11):1119-1126.

82. Van Raamsdonk CD, Bezrookove V, Green G, Bauer J, Gaugler L, O'Brien JM, Simpson EM, Barsh GS, Bastian BC: Frequent somatic mutations of GNAQ in uveal melanoma and blue naevi. Nature 2009, 457(7229):599-602.

83. Van Raamsdonk CD, Griewank KG, Crosby MB, Garrido MC, Vemula S, Wiesner T, Obenauf AC, Wackernagel W, Green G, Bouvier N, et al: Mutations in GNA11 in uveal melanoma. N Engl J Med 2010, 363(23):2191-2199.

84. Liu S, Umezu-Goto M, Murph M, Lu Y, Liu W, Zhang F, Yu S, Stephens LC, Cui $X$, Murrow $G$, et al: Expression of autotaxin and lysophosphatidic acid receptors increases mammary tumorigenesis, invasion, and metastases. Cancer Cell 2009, 15(6):539-550

85. Barker $\mathrm{N}$, Clevers $\mathrm{H}$ : Mining the Wnt pathway for cancer therapeutics. Nat Rev Drug Discov 2006, 5(12):997-1014.

86. Huang W, Lv X, Liu C, Zha Z, Zhang H, Jiang Y, Xiong Y, Lei QY, Guan KL: The $\mathrm{N}$-terminal phosphodegron targets TAZ/WWTR1 protein for SCFbeta-TrCPdependent degradation in response to phosphatidylinositol 3-kinase inhibition. J Biol Chem 2012, 287(31):26245-26253.

87. Konsavage WM, Yochum GS: Intersection of Hippo/YAP and Wnt/ $\beta$ catenin signaling pathways. Acta Biochim Biophys Sin 2013, 45(2):71-79.

88. Rosenbluh J, Nijhawan D, Cox AG, Li X, Neal JT, Schafer EJ, Zack TI, Wang X, Tsherniak A, Schinzel AC: $\beta$-Catenin-Driven Cancers Require a YAP1 Transcriptional Complex for Survival and Tumorigenesis. Cell 2013, 153(1):267-270.
89. Heallen T, Zhang M, Wang J, Bonilla-Claudio M, Klysik E, Johnson RL, Martin JF: Hippo pathway inhibits Wnt signaling to restrain cardiomyocyte proliferation and heart size. Science 2011, 332(6028):458-461.

90. Imajo M, Miyatake K, limura A, Miyamoto A, Nishida E: A molecular mechanism that links Hippo signalling to the inhibition of Wnt/ $\beta$-catenin signalling. EMBO J 2012, 31(5):1109-1122.

91. Varelas X, Miller BW, Sopko R, Song S, Gregorieff A, Fellouse FA, Sakuma R, Pawson T, Hunziker W, McNeill H, et al: The Hippo pathway regulates Wnt/ beta-catenin signaling. Dev Cell 2010, 18(4):579-591.

92. Li VS, Clevers H: Intestinal Regeneration: YAP_-Tumor Suppressor and Oncoprotein? Curr Biol 2013, 23(3):R110-R112.

doi:10.1186/2045-3701-3-33

Cite this article as: Guo and Zhao: Integration of mechanical and chemical signals by YAP and TAZ transcription coactivators. Cell \& Bioscience 2013 3:33.

\section{Submit your next manuscript to BioMed Central and take full advantage of:}

- Convenient online submission

- Thorough peer review

- No space constraints or color figure charges

- Immediate publication on acceptance

- Inclusion in PubMed, CAS, Scopus and Google Scholar

- Research which is freely available for redistribution 